\title{
Invited Discussion on: "Impact of Dorsal Preservation Rhinoplasty Versus Dorsal Hump Resection on the Internal Nasal Valve: A Quantitative Radiologic Study"
}

\author{
Robert H. Gilman ${ }^{1}$ (1)
}

Received: 30 January 2020/Accepted: 31 January 2020/Published online: 13 February 2020

(C) Springer Science+Business Media, LLC, part of Springer Nature and International Society of Aesthetic Plastic Surgery 2020

Level of Evidence $V$ This journal requires that authors assign a level of evidence to each article. For a full description of these Evidence-Based Medicine ratings, please refer to the Table of Contents or the online Instructions to Authors www.springer.com/00266.

I commend the authors on an interesting and wellwritten manuscript comparing effects of classic hump resection rhinoplasty versus dorsal preservation rhinoplasty on the internal nasal valve angle and cross-sectional area as measured radiographically. Dorsal preservation rhinoplasty (DPR) has become a hot topic in the last several years with a number of prominent proponent's worldwide [1-6]. Among the suggested advantages of DPR is the maintenance of the keystone area anatomy, thus eliminating the need for reconstruction of the middle cartilaginous vault after lowering the dorsum by classic hump resection rhinoplasty [7]. DPR decreases the possibility of medial disarticulation of the upper lateral cartilages from the nasal bones with the resulting inverted "V" deformity and also maintains and often improves the relationship of the upper and lower lateral cartilages preserving or improving the internal nasal valve. DPR may also preserve a more natural smooth appearance to the osteo-cartilaginous junction and better preserve natural dorsal aesthetic lines. The forces of the downward movement of the entire nasal pyramid tend to round out and open the angle of the "roof" at the internal valve. This maneuver should result in an increase

Robert H. Gilman

gilmanr@med.umich.edu

1 Section of Plastic Surgery, University of Michigan Medical Center, 2130 Taubman Center, SPC 5340, 1500 E Medical Center Dr., Ann Arbor, MI 48109, USA in the cross-sectional area of the internal nasal valve as well as an increase in the angle formed by the junction of the septum with the lateral nasal cartilage. Indeed, in tethered and over projecting noses with long nostrils, dorsal preservation can open the internal valve to the extent of causing flaring of the ala necessitating alar reduction [3].

Most of the discussions concerning the value of DPR in preserving the function of the internal nasal valve are based on subjective observations of changes at this level during and after DPR. Some further evidence of DPR aiding internal valve function comes from limited use of PROMs such as the 10-item Standardized Cosmesis and Health Nasal Outcomes Survey (SCHNOS) for functional and cosmetic rhinoplasty [8]. To date, there have not been objective studies which actually measure the postoperative changes in the area of the internal nasal valve. This study is the first to do so by measuring pre-procedure both the cross-sectional area at the internal nasal valve and the change in the internal nasal valve angle and comparing these measurements after conventional hump resection, push-down (PD) technique and let-down (LD) technique.

The study utilized three cadaver heads for each of the three techniques for hump reduction. The same heads were used for the PD and LD techniques as the only difference between the two was the removal of a wedge of bone from the lateral nasal walls for the LD technique. Measurements of the internal nasal valve were taken after the PD technique, and then, the segment was disimpacted, bony wedges were removed, the LD technique was completed and the internal nasal valve area was measured. Measurement of the cross-sectional area and the internal nasal valve angle were done utilizing CT scans.

The authors found that there was no appreciable narrowing of the internal nasal valve angle or cross-sectional 
area in both the classic hump reduction with spreader grafts or flaps and the LD technique while there was significant narrowing in the internal valve angle and cross-sectional area in the PD technique. The authors attribute the internal nasal valve compromise to the required medial pinching of the lateral nasal walls necessary to impact the dorsal segment into the pyriform aperture. This pinching transmits to the internal valve area diminishing both the internal nasal valve angle and the cross-sectional area.

Previous studies have used acoustic rhinomanometry to measure internal valve function in cadaveric specimens receiving conventional hump resection without spreader grafts or flaps [9]. There are no studies to my knowledge that assess the function of the internal valve in a rigorous and reproducible manner in patients undergoing DPR by either PD or LD. The authors acknowledge the need for these kinds of studies in patients. This study would have benefitted from a larger cadaveric sample. In addition, the re-use three heads for the LD technique after disimpacting the segment from the PD technique have the potential to have affected the result.

The concept of DPR has been around for over a century and has been utilized as a procedure with varying degrees of enthusiasm for almost 70 years [10-12]. Enthusiasm for this procedure would seem to be rising since the millennium with a growing number of publications and courses being offered worldwide [1]. In his editorial, Rollin Daniel traced "generational epochs" in the evolution of rhinoplasty surgery beginning with Joseph as the father of modern rhinoplasty through Sheen who made rhinoplasty a truly aesthetic operation to the championing of open rhinoplasty by Gunter among others [2]. He points out the all too often need for complex revision rhinoplasty after conservative hump removal by traditional dorsal techniques necessitating often complex middle vault reconstruction. He cites preservation rhinoplasty as the next great revolution in rhinoplasty stating that this technique "replaces resection with preservation, excision with manipulation and secondary rib reconstruction with minimal revisions."

Whether Dr. Daniel and other prominent proponents of DPR prove to be correct is yet to be determined. One cannot help but being impressed watching a skilled surgeon perform this procedure and realizing that the keystone area is in fact an osteo-cartilaginous joint which can be manipulated and shaped without disrupting its function and without the need for post-reduction reconstruction. The promise of a smoother more natural dorsum, maintenance of dorsal aesthetic lines, faster recovery with less swelling and easier correction of unwanted postoperative irregularities is certainly enticing.

On the other hand, not every rhinoplasty surgeon feels the need to abandon the techniques that they have taken a career to hone with no guarantee that DPR will become the holy grail of rhinoplasty. Done thoughtfully, with meticulous attention to detail and planning, conservative hump reduction rhinoplasty with spreader flaps and grafts and careful middle vault reconstitution is still the overwhelming choice among rhinoplasty surgeons [13]. Being a rhinoplasty surgeon is much like being a chess master. The operation requires a great deal of forethought. Many if not most of the technical maneuvers that we do to create a specific desired change carry the potential to affect unwanted change. Understanding this dynamic takes time and experience [14].

I encourage those interested in rhinoplasty to read about, think about and spend time observing a surgeon experienced in DPR and then decide for yourself whether it is a technique you wish to learn and use. In the meantime, the authors of this manuscript have added information that can help us decide.

\section{Compliance with Ethical Standards}

Conflict of interest The author declares that he has no conflicts of interest to disclose.

Human and Animal Rights This article does not contain any studies with human participants or animals performed by the author.

\section{References}

1. Daniel RK, Saban Y, Cakir B, Palhazi P (2018) Course introduction from the faculty of Preservation Rhinoplasty Course, Istanbul

2. Daniel RK (2018) The preservation rhinoplasty: a new rhinoplasty revolution. Aesthet Surg J 38(2):228-229

3. Saban Y, Daniel RK, Polselli R, Trapasso M, Palhazi P (2018) Dorsal preservation: the push down technique reassessed. Aesthet Surg J 38(2):117-131

4. Boulanger N, Baumann C, Beurton R (2013) Septorhinoplasty by disarticulation: early assessment of a new technique for morphological correction of crooked noses. Rhinology 51(1):77-87

5. Ishida J, Ishida LC, Ishida H, Viera JC, Ferreira MC (1999) Treatment of the nasal hump with preservation of the cartilaginous framework. Plast Reconstr Surg 103(6):1729-1733

6. Montes-Bracchini JJ (2019) Nasal profile reduction using the LetDown Technique. Facial Plast Surg 35(5):486-491

7. Palhazi P, Daniel RK, Kosins AM (2015) The osseocartilaginous vault of the nose: anatomy and surgical observations. Aesthet Surg J 35(3):242-251

8. Moubayed SP, Ioannidis JPA, Saltychev M, Most SP (2018) The 10-item Standardized Cosmesis and Health Nasal Outcomes Survey (SCHNOS) for functional and cosmetic Rhinoplasty. JAMA Facial Plast Surg 20(1):37-42

9. Grymer LF (1995) Reduction rhinoplasty and nasal patency: change in the cross-sectional area of the nose evaluated by acoustic rhinometry. Laryngoscope 105(4 Pt 1):429-431

10. Lothrop OA (1914) An operation for correcting the aquiline nasal deformity; the use of new instrument; report of a case. Boston Sed Surg J 170:835-837

11. Cottle MH, Loring RM (1946) Corrective surgery of the external nasal pyramid and the nasal septum for restoration of normal physiology. Ill Med J 90:119-135 
12. Cottle MH (1954) Nasal roof repair and hump removal AMA. Arch Otolaryngol 60(4):408-414

13. Rohrich RJ, Muzaffar AR, Janis JE (2004) Component dorsal hump reduction: the importance of maintaining dorsal aesthetic lines in rhinoplasty. Plast Reconstr Surg 114(5):1298-1308

14. Constantian MB, Martin JP (2015) Why can't more good surgeons learn rhinoplasty? Aesthet Surg J 35(4):486-489
Publisher's Note Springer Nature remains neutral with regard to jurisdictional claims in published maps and institutional affiliations. 